\title{
Systematic Imaging Module in Complete Hindgut Duplication
}

\author{
Ashish Verma ${ }^{1}$ Prashant Nath Gupta ${ }^{1} \quad$ Vaibhav Pandey $^{2} \quad$ Shivi Jain $^{1} \quad$ Ashish Upadhyay $^{1}$ \\ jitendra Sharma ${ }^{1}$ Ram C. Shukla ${ }^{1}$ \\ ${ }^{1}$ Department of Radiodiagnosis and Imaging, Institute of Medical \\ Address for correspondence Ashish Verma, Department of \\ Sciences, Banaras Hindu University, Varanasi, India \\ Radiodiagnosis and Imaging, Institute of Medical Sciences, Banaras \\ 2 Department of Pediatric Surgery, Institute of Medical Sciences, \\ Banaras Hindu University, Varanasi, India \\ Hindu University, University Road, Varanasi, Uttar Pradesh 221005 \\ India (e-mail: drdnv5@gmail.com).
}

Eur J Pediatr Surg Rep 2015;3:50-53.

\begin{abstract}
Keywords

- hindgut duplication

- barium enema

- MRI

Complete hind gut and anal canal duplication is a rare entity, usually remaining asymptomatic till the disease comes to light due to associated anomalies or due to cosmetic reasons. Classical imaging consisting of barium enema examination served a limited role, in terms of depicting the length of gut segment involved. Technical advances in magnetic resonance imaging (MRI) with three-dimensional (3D) reformations cannot only solve the above purpose but further evaluate key points needed for surgical planning. The present technical report lays out a systematic module for evaluation of various aspects of complete hindgut duplication, critical for management. The role of 3D MRI is emphasized upon, for evaluation of pelvic floor and anorectum, even in infants with a distorted anatomy.
\end{abstract}

\section{Introduction}

Duplication of the gut is an uncommon entity, most commonly involving the small bowel (60\% of the cases). ${ }^{1}$ Large bowel duplication is quite rare with only few case reports present in the literature. The cecum is a part involved in most instances, with only 4 to $18 \%$ of the cases having a colonic duplication. ${ }^{2}$ Three-fourth of all the cases of colonic duplication have a duplication cyst at the mesenteric border while one-fourth have a tubular duplicated moiety which may or may not communicate with the adjacent native bowel. ${ }^{3}$ In either scenario, most cases remain asymptomatic, however a larger subset of cases of tubular duplication present with pain and obstruction, ${ }^{4}$ as a result of the combined effect of poor propulsive action of bowel muscularis and formation of fecoliths in either moiety. ${ }^{5}$ In cases of tubular duplication, diagnosis is mostly evident on clinical grounds as both moieties have a perineal communication. The role of imaging was as yet, restricted to deciphering the length of duplicated segments and their intercommunication, depicted on contrast enema

received

September 27, 2014 accepted after revision December 16, 2014

published online

March 10, 2015 radiography. ${ }^{6}$ Cross-sectional imaging has however added a new dimension to presurgical evaluation of the pathology by providing information about adequacy of mural architecture of bowel and that of pelvic floor musculature. ${ }^{7}$ In the presented rare case of communicating tubular duplication of hindgut, a systematic imaging algorithm assisted our surgical team to logically plan a corrective surgery and achieve good postoperative colonic functionality with adequate continence.

\section{Case Report}

A 9-month-old male child presented to the pediatric surgery emergency with acute abdomen. In addition the parents reported presence of two anal openings, with intermittent passage of fecal material through either opening. A diagnosis of hindgut duplication was quite evident and, in view of the presenting symptom an invertogram was done to assess the duplicated anorectum ( - Fig. 1a). The radiograph however could not help much as no air was seen in either moiety. Further, a contrast enema study ( - Fig. 1 b) was done which
License terms Stuttgart · New York

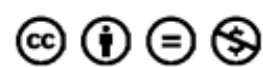




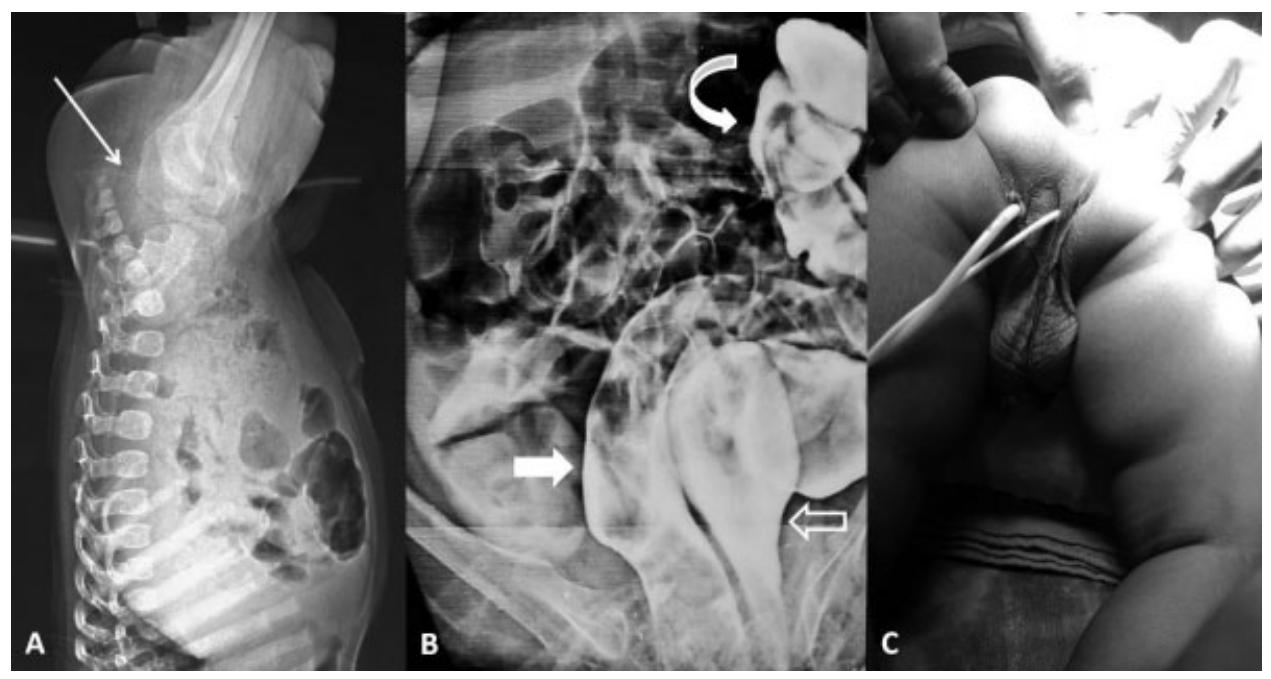

Fig. 1 (A) Invertogram done during initial presentation to evaluate the lower gastrointestinal tract shows no air in the anorectum (straight arrow). (B) Contrast enema radiography done with thin barium shows the relatively smaller (solid arrow) and larger (hollow arrow) moiety of duplicated descending colon, uniting at the splenic flexure (curved arrow). (C) Clinical picture showing passage of two catheters per rectum, through two different anal openings.

revealed duplication of anorectum until the splenic flexure with fusion of the two moieties thereby to form a single transverse colon. The moieties followed a parallel course and were located at the expected anatomical location. Haustrations were noted in both moieties with fecoliths seen in the smaller moiety located medially (which probably was the cause of obstruction). Also fewer peristaltic waves were seen in the smaller moiety (on fluoroscopy), both findings confirmed the diagnosis, hence corrective surgery consisting of resection of the dividing septum was planned. A suggestion was made from the end of our pediatric imaging unit, in favor of a preoperative magnetic resonance imaging (MRI), in view of an alternative approach for correction (i.e., resection of the lesser moiety) documented in the literature. The latter technique is based on presence of adequate mural architecture of the greater (larger) moiety with a single pelvic diaphragm. MRI was done on a 1.5 T superconducting magnet using a body array coil using a "differential contrast technique" (i.e., after instillation of air in lesser and water in greater moiety using a soft balloon catheter). The imaging

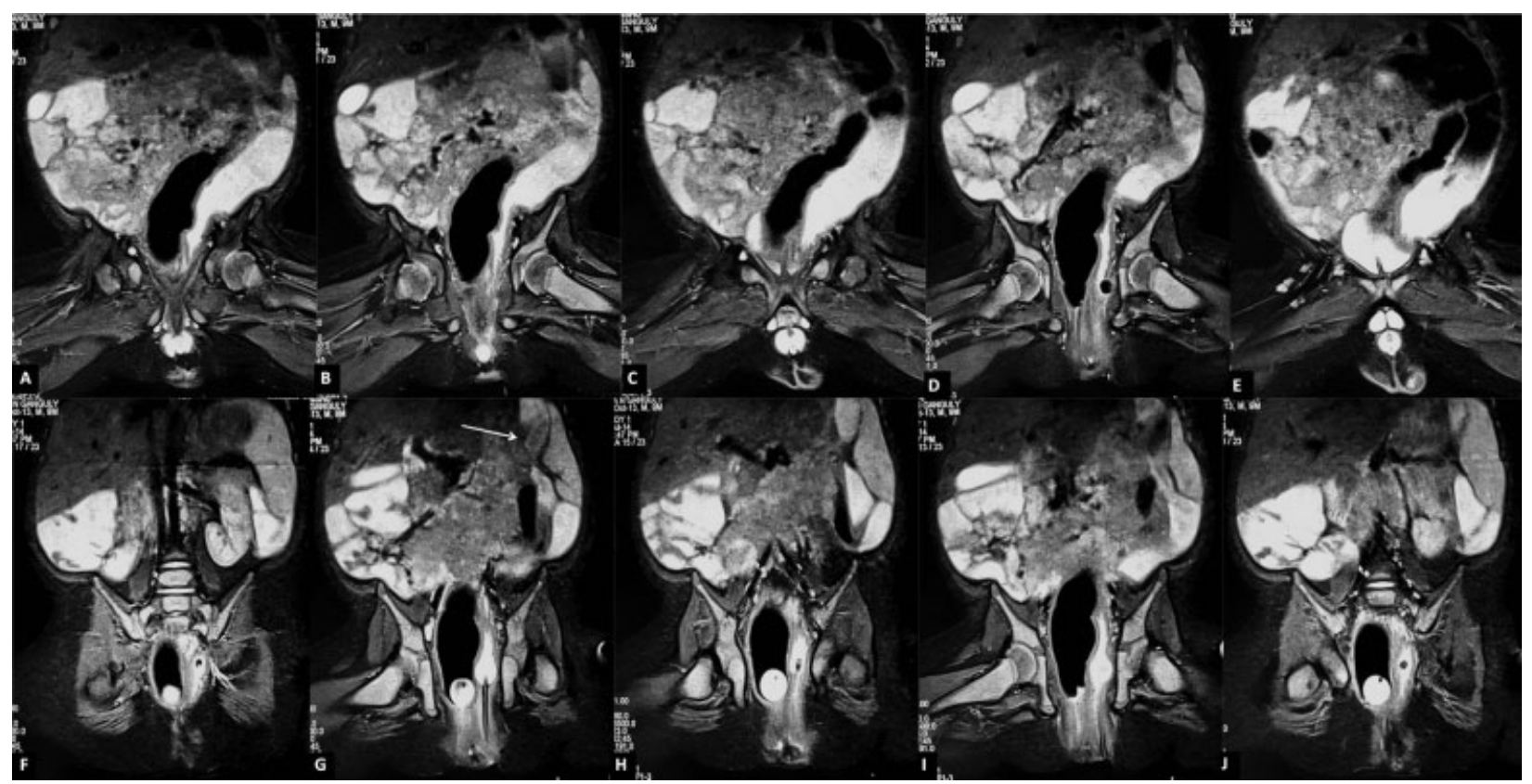

Fig. 2 Serial coronal T2-weighted magnetic resonance images (from a three-dimensional stack) from anterior to posterior (A-J) performed with differential contrast technique shows the two moieties of duplicated descending colon, the medial moiety was insufflated with air while the lateral was filled with saline. Note the point of union at the splenic flexure (straight arrow in G). Note the clear separation of the wall of both the moieties with a clear cut plane between the two. 


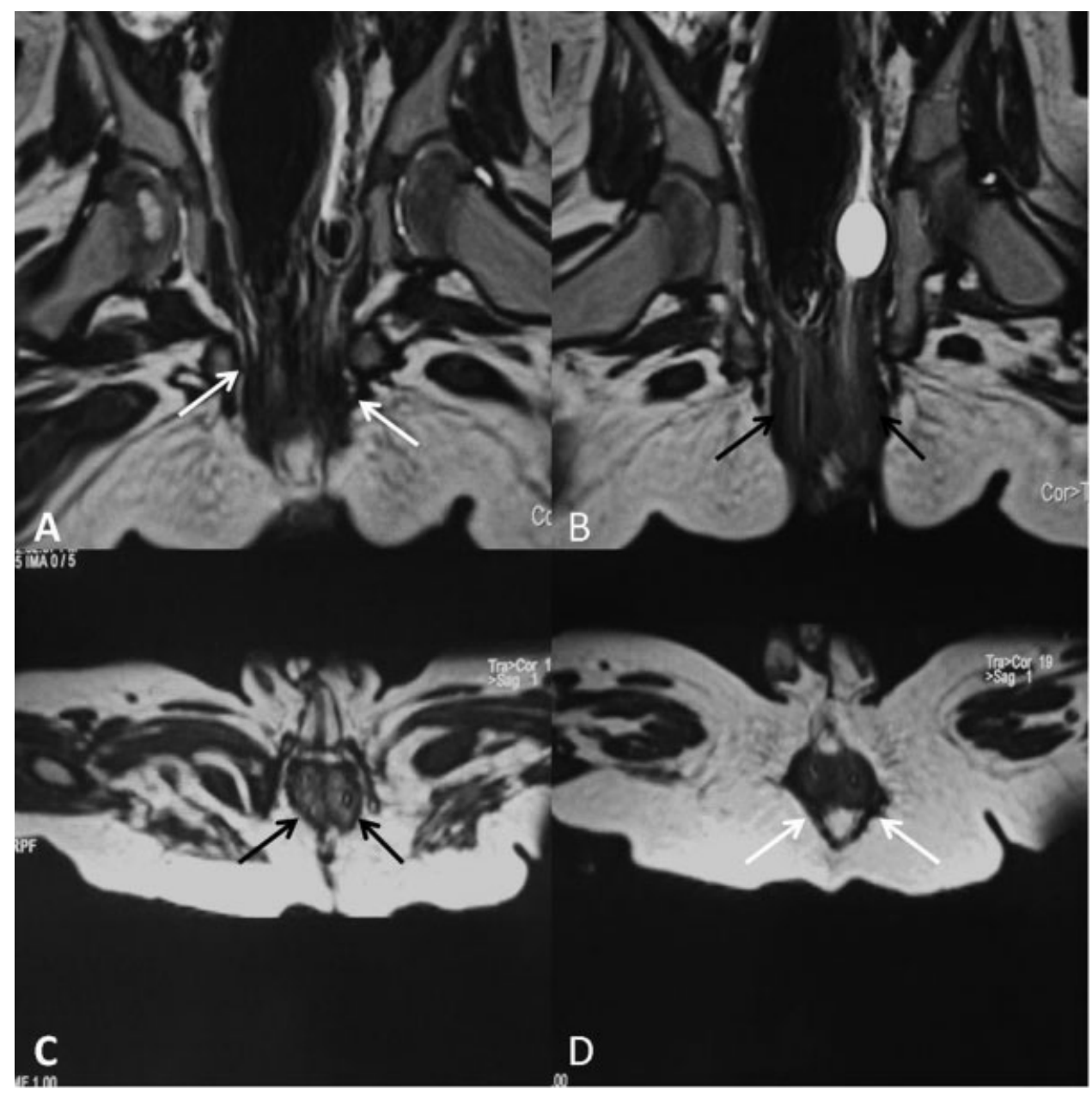

Fig. 3 Coronal (A, B) and axial (C, D) reformatted 3D T2-weighted images (from a 3D stack) showing the internal (black arrows) and external sphincters (white arrows), having a normal anatomy and continuity. 3D, three-dimensional.

confirmed the findings noted on the contrast enema radiography ( - Fig. 2 ), further both the moieties were found to have equal and well developed mural architecture consisting of circular and longitudinal muscle layers (-Figs. 2 and 3). Focused imaging of the pelvis revealed the anatomy of the pelvic floor to be normal and adequate, the concentric puborectal sling was single with absence of any pelvic floor fibers decussating between the two moieties. The longitudinal muscles in the anorectal region were continuous with no atrophy or fat infiltration within or around the sphincters. No urinary bladder duplication was noted in this case. A posterior sagittal anorectoplasty (PSARP) assisted by laparotomy was performed in view of the high position of the distal end of the proximal loop. Surgical resection of the lesser moiety (-Fig. 4) was done with closure of distal opening. Dissection was done to carefully extract the lower end of lesser moiety from the pelvic sling, such that the greater moiety remained finally encircled by the native muscles of pelvic diaphragm. Strengthening of the sphincter and levator ani was done in addition. The procedure remained uneventful and the postoperative course was satisfactory. Passage of flatus and fecal matter was noted at the 7 th hour after initiation of oral intake on the 5th day. Development of continence, appropriate for the age was noted on follow-up visit at 3 years using the Kelly score. $^{8}$

\section{Discussion}

Surgical correction of duplicated hindgut is reserved exclusively for patients presenting with symptoms such as acute abdomen secondary to obstruction, intussusceptions or bleeding per rectum. ${ }^{9}$ This consists primarily of excision of the duplicated moiety in most instances. ${ }^{10}$ Alternatively division of the intervening septum (for tubular duplication) or cyst marsupialization (for duplication cyst) combined with mucosal stripping of the remaining cyst may be done. ${ }^{11}$ Though the latter techniques carry less satisfactory results, in terms of recurrence of symptoms, they are preferred by some in view of the lower incidence of operative complications with them. ${ }^{12}$ The major problems described above with the former technique include postoperative hemorrhage due to remaining bowel ischemia secondary to inadvertent removal of common mural components with common vascular connection and, continence issues due to injury to intervening decussating pelvic diaphragm components. ${ }^{13-15}$ These are precisely the questions that we ensured to address by MRI in the present 


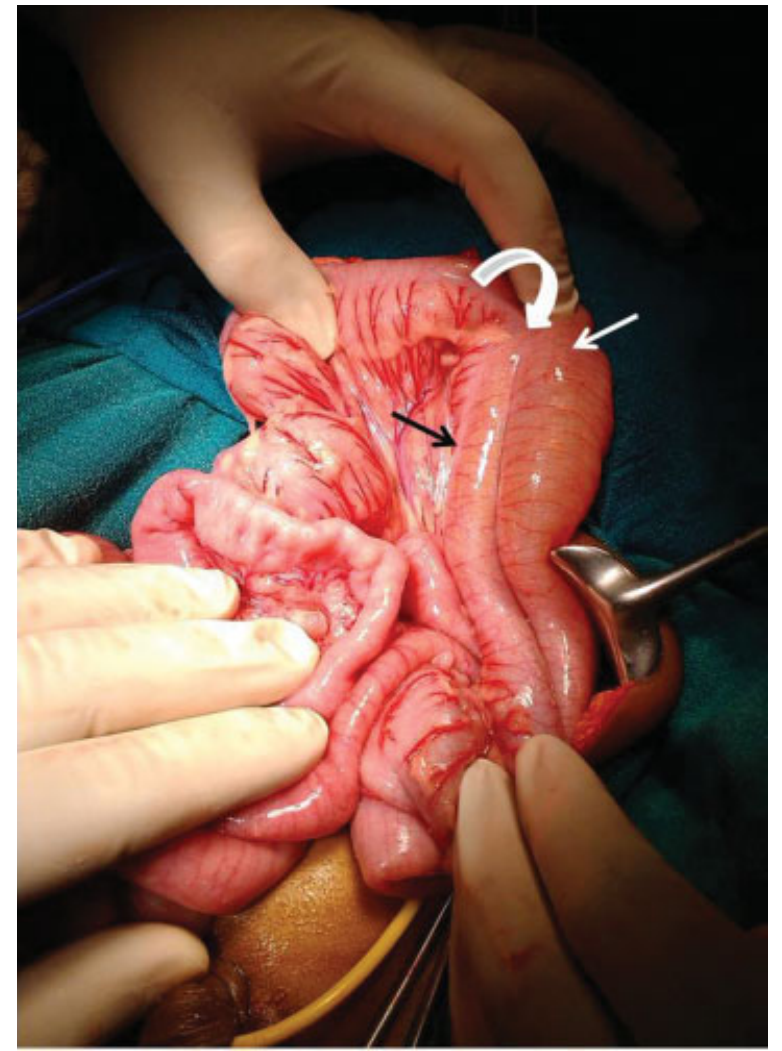

Fig. 4 Operative image showing the smaller (black arrow) and the larger (white arrow) moieties of duplicated descending colon uniting at the splenic flexure (curved arrow). Note the separate vasculature of both moieties entering separately from the mesenteric border.

case, whereby both the moieties had a separate and adequate mural architecture with a clear cut plane of separation between the two ( - Figs. 2 and 3). This ensured no vascular crossconnections, an injury to which could have led to precipitous postoperative primary hemorrhage, as well as delayed bleeding due to ischemia of the preserved bowel loop. Further, exclusion of a muscular connection between the loops made the surgeon confident of excising the lesser moiety without any chance of postoperative fecal leak or delayed pseudodiverticula formation. MRI has been advocated as the most suitable technique to evaluate the pelvic floor structures at all ages and for all indications, with endorectal sonography being the only other competing modality. ${ }^{16,17}$ In pediatric age group however, MRI remains the investigation of choice for the purpose as pediatric endorectal probes are not available commonly (and were not available with us as well), also there is greater operator dependence and interobserver variability as far as endorectal sonographic evaluation of pelvic floor is concerned. ${ }^{17}$ The sphincteric fibers (puborectalis) and the fibers of pelvic diaphragm develop from the local mesoderm, simultaneous to the developing ectodermal anorectum which finally fuse to form a continuous channel. ${ }^{4}$ Duplication would permit the mesenchymal tissue to interdecussate between the two moieties, leading to chances of an inadvertent injury to these fibers if not detected preoperatively. MRI excluded the presence of any such redundant intervening tissue, an injury to which could have caused continence issues ( - Fig. 3 ). Further detection of an associated malformations such as bladder duplication, spinal dysraphism, anorectal malformation (not seen in this case), by MRI, can enable one to plan concomitant corrections. $^{4}$

Though hindgut duplication has been reported previously, the present reports aims at providing an insight to the imaging expert, as to the crucial role he can play in systematic management of this rare and intriguing clinical entity. Conventional contrast enema radiography remains the mainstay for initial evaluation of the type and length of duplication. Preoperative MRI should be performed in all cases to rule out associated malformations and to more precisely define the anatomy before surgery.

\section{References}

1 Fotiadis C, Genetzakis M, Papandreou I, Misiakos EP, Agapitos E, Zografos GC. Colonic duplication in adults: report of two cases presenting with rectal bleeding. World J Gastroenterol 2005; 11(32):5072-5074

2 Macpherson RI. Gastrointestinal tract duplications: clinical, pathologic, etiologic, and radiologic considerations. Radiographics 1993;13(5):1063-1080

3 Sengar M, Gupta CR, Jain V, Mohta A. Colorectal duplication with prostatorectal fistulae. J Pediatr Surg 2013;48(4):869-872

4 Singh S, Ahmed I, Rawat J, Panday A. Association of anorectal malformation with duplicated colon, sacral meningomyelocele and scrotal anomalies. BMJ Case Rep 2011;pii:bcr1220103632

5 Khaleghnejad Tabari A, Mirshemirani A, Khaleghnejad Tabari N. Complete colonic duplication in children. Caspian J Intern Med 2012;3(2):436-439

6 Okoro PE, Obiorah C. Duplication cyst of the bowel causing ileal volvulus. A case report. Niger J Med 2010;19(2):230-232

7 Puligandla PS, Nguyen LT, St-Vil D, et al. Gastrointestinal duplications. J Pediatr Surg 2003;38(5):740-744

8 Kelly JH. The clinical and radiological assessment of anal continence in childhood. Aust N Z J Surg 1972;42(1):62-63

9 Dombale V, Patil BV, Kadam SA, Kerudi BH. Enteric duplication cyst of cecum presenting with intestinalobstruction - A case report. JKIMSU 2012;1(2):147-149

10 Holcomb GW III, Gheissari A, O’Neill JA Jr, Shorter NA, Bishop HC. Surgical management of alimentary tract duplications. Ann Surg 1989;209(2):167-174

11 Pruthi S, Parisi MT. 'Double trouble': incidental diagnosis of colonic duplication on spinal MRI. Pediatr Radiol 2008;38(10):1143

12 Van Elst F, Hubens A. Duplication of the colon in the adult (author's transl) [in Dutch]. Acta Chir Belg 1978;77(5):335-342

13 Frittelli P, Costa G, Zanella L, Sguazzini G, Rossi FS. Intestinal duplication in the adult. A case report of colonic duplication and a review of the literature [in Italian]. Chir Ital 2002;54(5): 721-728

14 Salvador II, Modelli ME, Pereira CR. Tubular duplication of the colon: a case report and review of the literature [in Portuguese]. J Pediatr (Rio J) 1996;72(4):254-257

15 O'Neil J, Rowe M. Duplications of the gastrointestinal tract. In: Essentials of Pediatric Surgery. St. Louis, MO: Mosby Yearbook; 1995:520-525

16 Law YM, Fielding JR. MRI of pelvic floor dysfunction: review. AJR Am J Roentgenol 2008;191(6, Suppl):S45-S53

17 Engin G. Endosonographic imaging of anorectal diseases. J Ultrasound Med 2006;25(1):57-73 